\title{
modèle de comportement des sols non cohérents
}

\author{
a constitutive model for non-cohesive soils
}

\author{
B. CAMBOU - K. JAFARI
}

Ecole Centrale de Lyon

GRECO - CNRS - Rhéologie des Géomatériaux*

Rev. Franç. Géotech. n 44, p.p. $43-55$ (juillet 1988)

\section{Résumé}

L'article présente un modèle de comportement des sols non cohérents bien adapté, en particulier, au cas de sollicitations complexes (cycliques, avec rotation des directions principales). Après avoir défini le cadre thermodynamique général (élasto-plasticité, matériaux standard généralisés), les mécanismes de déformations plastiques isotropes et déviatoires sont présentés. le couplage entre ces deux mécanismes est décrit par une condition cinématique. La technique d'identification du modèle est ensuite présentée, enfin la dernière partie est consacrée à la présentation de prévisions et à leur comparaison avec les résultats expérimentaux pour la plupart inconnus lors de la prévision théorique.

\begin{abstract}
This work presents a modelisation of granular materials behavior well adapted to complex loadings (cyclic, with rotation of principal axes). After defining the general thermodynamic framework, the isotropic and deviatoric yielding mechanisms are developed. The coupling between these two mechanisms is described by a kinematic condition. Then the calibration of the model is defined and at last theoretical predictions are presented and compared with experimental results.
\end{abstract}




\section{INTRODUCTION}

\subsection{Type du modèle proposé}

L'apparition de la méthode des éléments finis ainsi que d'ordinateurs de plus en plus puissants et rapides a permis le développement de codes de calcul de plus en plus élaborés. Toutefois l'efficacité de ces codes est en grande partie liée à la validité de la loi de comportement utilisée. Ces dernières années de nombreux modèles ont été proposés dans la littérature et l'utilisateur peut parfois être dérouté par cette multiplicité. Cette diversité s'explique car après avoir rêvé au modèle universel donnant réponse à tout, les spécialistes semblent actuellement s'accorder sur la définition de grandes classes de modèles chacune étant bien adaptée à la résolution de certains types de problèmes. Il apparaît donc fondamental de définir de façon claire le domaine de validité de chaque modèle, ainsi que le degré de complexité de son identification.

Le modèle que nous présentons est bien adapté à la description du comportement des matériaux non cohérents soumis à des sollicitations complexes: avec rotation des directions principales, cycliques (avec un faible nombre de cycles, la qualité des prévisions n'a pas été analysée au-delà).

L'identification expérimentale du modèle nécessite la détermination de 12 paramètres: parmi ceux-ci 8 sont faciles à déterminer, les 4 derniers sont de détermination plus délicate. Cette identification se fait à partir d'essais triaxiaux classiques et isotropes.

\subsection{La réalité expérimentale et le cadre général de la modélisation}

Les résultats expérimentaux observés sur les sables montrent :

- l'existence de déformations réversibles et irréversibles sous sollicitations isotropes et déviatoires (fig. 1, 2);

- que ces déformations (réversibles et irréversibles) évoluent de façon non linéaire en fonction de la sollicitation (fig. 1, 2);

- que sous une sollicitation déviatoire il apparaît des variations de volume (domaine de contractance et domaine de dilatance séparés par une surface d'état caractéristique (LUONG 1980) (fig. 2) ;

- que le critère de rupture dépend du troisième invariant du tenseur des contraintes (fig. 3);

- que toute sollicitation déviatoire fait apparaître une très forte anisotropie induite (fig. 4);

- que pour un nombre de cycles limité on observe toujours une évolution des déformations irréversibles, il n'y a pas accommodation des cycles.

Pour décrire cette réalité expérimentale nous avons choisi une modélisation élasto-plastique en nous plaçant quand cela était possible dans le cadre thermodynamique des matériaux standard généralisés.

Dans ce cadre général nous avons développé les éléments de modélisation suivants:

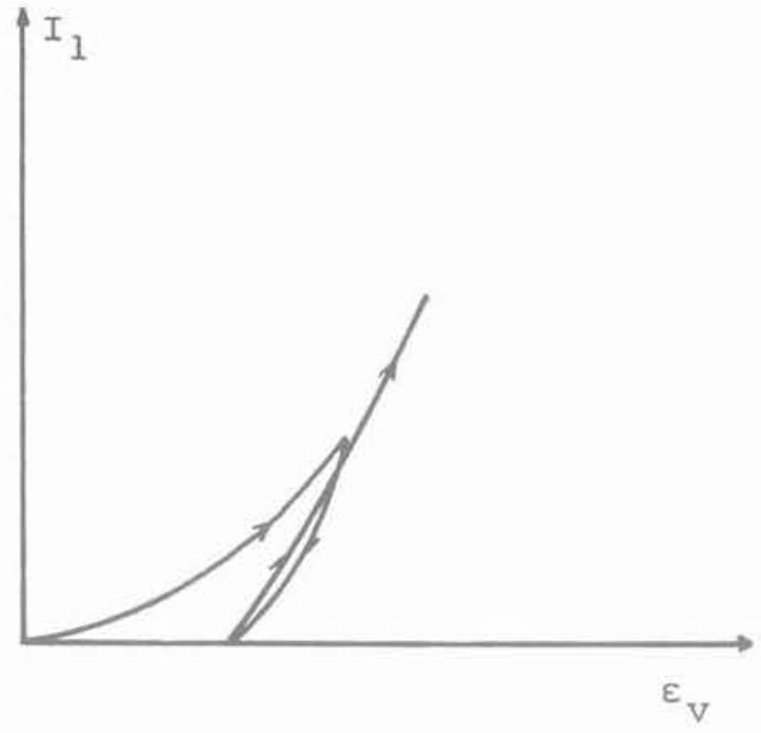

Fig. 1. - Essai triaxial isotrope.

Fig. 1. - Hydrostatic loading and unloading.

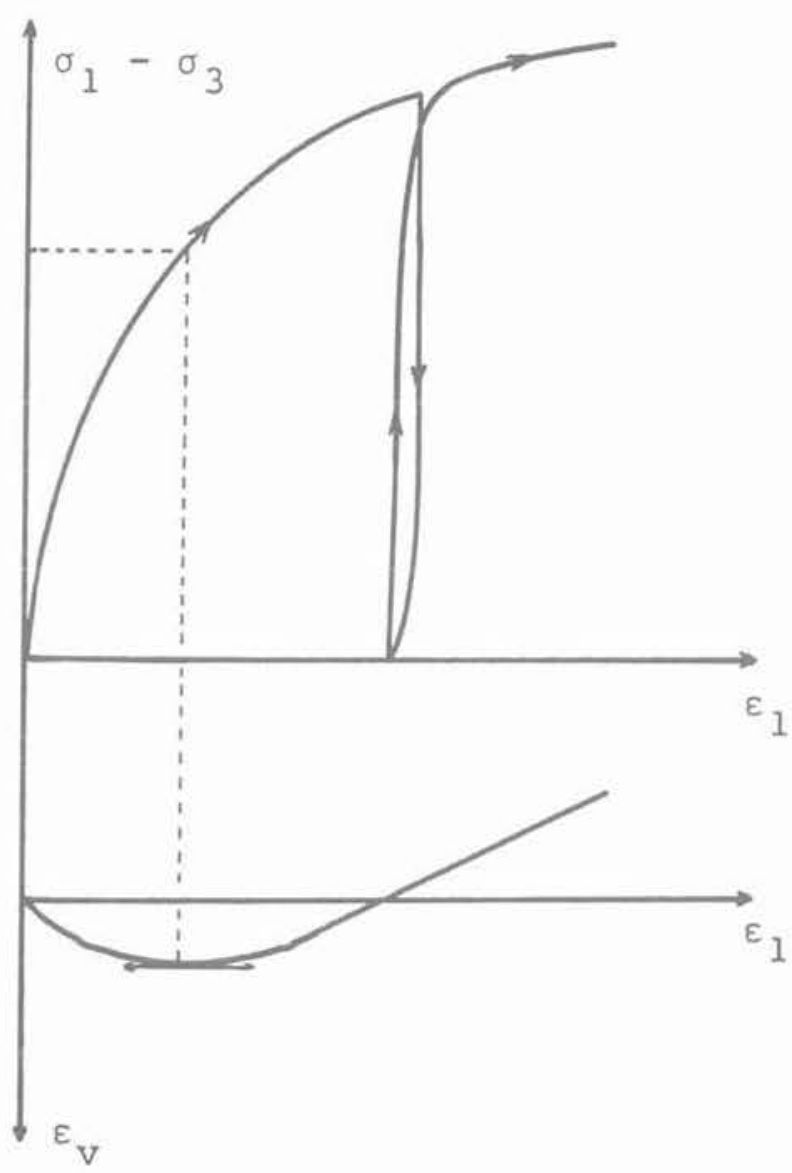

Fig. 2. - Essai triaxial axisymétrique.

Fig. 2. - Axisymetric triaxial test (loading and unloading). 


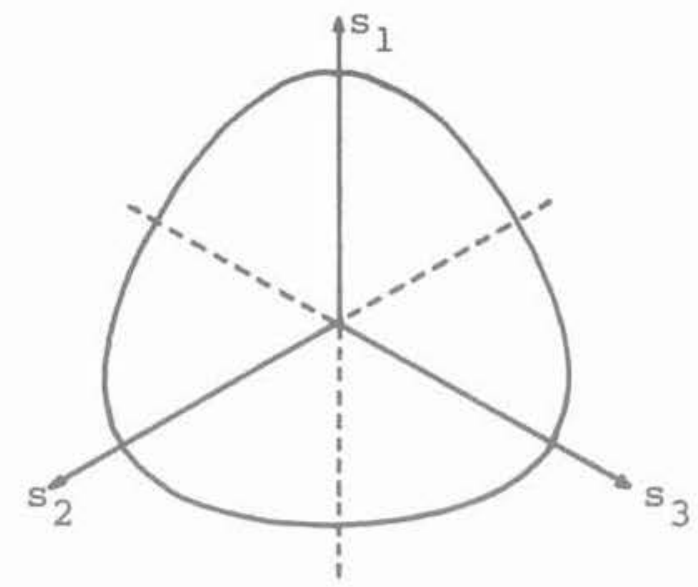

Fig. 3. - Forme du critère de plasticité dans le plan déviatoire.

Fig. 3. - Shape of the plasticity criterion in the deviatoric plane.

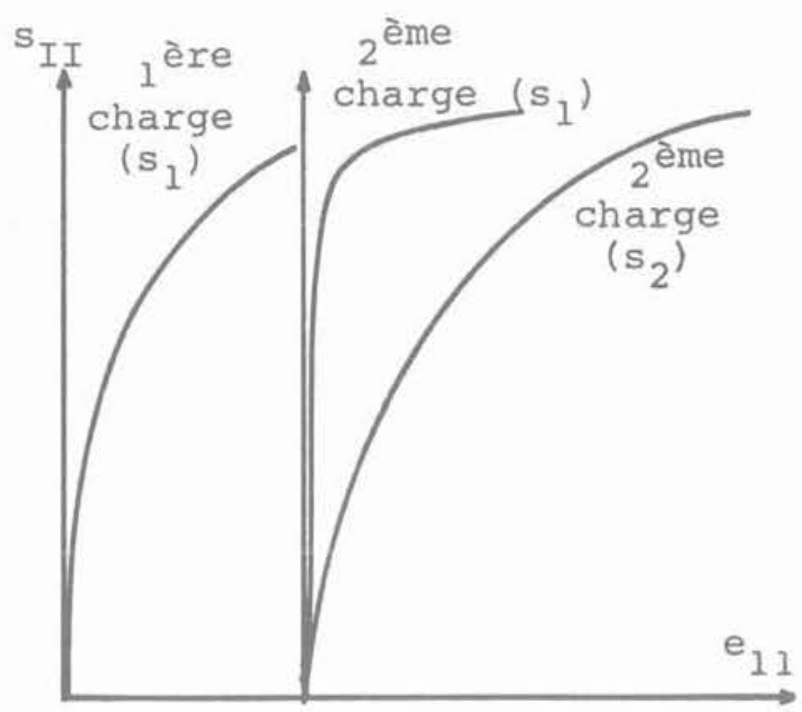

Fig. 4. - Mise en évidence à l'appareil triaxial véritable de l'anisotropie induite (d'après des essais réalisés par J. Lanier, I.M.G. Grenoble).

Fig. 4. - Thrue triaxial test results showing an important induced anisotropy (from results obtained by J Lanier IMG Grenoble).

- une élasticité non linéaire pour la description des déformations réversibles ;

- un mécanisme de déformation plastique $\left(\varepsilon_{v}^{\text {p }}\right)$ lié à la variation de la partie isotrope du tenseur des contraintes $\left(\mathrm{I}_{1}\right)$ dont l'évolution est définie par un écrouissage isotrope :

- un mécanisme de déformation plastique $\left(\varepsilon^{\mathrm{dp}}\right)$ lié à la variation du déviateur rapporté à la contrainte moyenne $\left(\mathrm{s}_{4} / \mathrm{I}_{1}\right)$ dont l'évolution est définie par 2 écrouissages, l'un isotrope, l'autre cinématique;

- une condition cinématique permettant une modélisation correcte des variations de volume.
Nous présenterons donc chacun de ces éléments de modélisation, puis la technique d'identification du modèle ainsi que des résultats de simulations dont la plupart ont été réalisées sans avoir connaissance des résultats expérimentaux.

Les principales notations utilisées dans cet article sont définies ci-dessous :

$\sigma_{0}:$ Tenseur des contraintes

$s_{v}$ : Partie déviatoire du tenseur des contraintes

$I_{1}:$ Premier invariant de $\sigma_{0}$

$\mathrm{s}_{\mathrm{t}}$ : Deuxième invariant de $\mathrm{s}_{\mathrm{i}}$

$\varepsilon_{0}:$ Tenseur des déformations

$\varepsilon_{0}^{e}$ : Partie élastique de $\varepsilon_{0}$

$\varepsilon_{\mathrm{v}}^{\mathrm{p}}$ : Partie plastique de $\varepsilon_{\mathrm{i}}$

$\varepsilon_{v}^{\text {tp }}$ : Déformation plastique volumique liée à la variation de la partie isotrope des contraintes $I_{1}$.

$\varepsilon^{\text {dp }}$ : Déformation plastique liée à la variation du déviateur des contraintes rapporté à la contrainte moyenne $\left(\mathrm{s}_{\mathrm{v}} / \mathrm{l}_{1}\right)$

$e^{d p}$ : Partie déviatoire de $\varepsilon^{d p}{ }_{i j}$

$\varepsilon^{d p_{p}}$ : Déformation volumique dans $\varepsilon^{d p}{ }^{d \|}=\varepsilon^{d p}$

\section{QUELQUES NOTIONS SUR LE FORMALISME DES MATÉRIAUX STANDARD GÉNÉRALISÉS}

Les matériaux standards généralisés (MSG) ont été introduits par HALPHEN et NGUYEN (HALPHEN et NGUYEN 1975). Ce formalisme a été développé dans le cadre thermodynamique des processus irréversibles.

La dissipation est définie à partir de l'inégalité de CLAUSIUS - DUHEM

$$
\varnothing=\sigma_{0} \dot{\varepsilon}_{j}-\dot{w} \geqslant 0
$$

w est l'énergie stockée qui dépend des variables d'état (mesurables ह ou cachées $\underline{\underline{p}}^{p}, \mathrm{x}_{\mathrm{k}}$ ):

$$
w=\underline{\underline{\underline{E}}} \underline{\varepsilon}^{e}: \underline{\underline{\varepsilon}}^{e}+w\left(x_{k}\right)
$$

avec :

$$
\underline{\varepsilon}^{e}=\underline{\varepsilon}-\underline{\underline{\varepsilon}}
$$

$x_{k}$ représentent tous les paramètres d'écrouissage scalaires ou tensoriels.

La dissipation peut alors s'écrire:

$$
\begin{aligned}
\emptyset=\left(\underline{\underline{g}}-\frac{\partial \mathrm{w}}{\partial \underline{\underline{\varepsilon}}}\right): \stackrel{\varepsilon}{\varepsilon}^{e}+\underline{\underline{\sigma}}: \underline{\underline{\varepsilon}^{p}} & \\
& -\frac{\partial \mathrm{w}}{\partial \mathrm{x}_{k}} \mathrm{x}_{k} \geqslant 0
\end{aligned}
$$


La déformation élastique n'introduisant aucune dissipation le premier terme doit s'annuler donc:

$$
\begin{gathered}
\underline{\sigma}=\frac{\partial w}{\partial \underline{\underline{\varepsilon^{e}}}}=\underline{\underline{\underline{\underline{E}}}} \underline{\underline{\underline{\varepsilon^{e}}}} \\
\emptyset=\underline{\underline{g}}: \underline{\underline{\varepsilon}}^{p}-\mathrm{X}_{\mathrm{k}} \dot{\mathrm{x}}_{\mathrm{k}} \geqslant 0
\end{gathered}
$$

avec $\mathrm{X}_{k}=\frac{\partial \mathrm{w}}{\partial \mathrm{x}_{\mathrm{k}}}$ définissant la force thermodynamique associée à $x_{k}$

L'équation (5) permet de définir les lois d'évolution des variables flux thermodynamiques $\left(\varepsilon^{p}, x_{k}\right)$ en dérivant la fonction de charge $f$ par rapport aux forces thermodynamiques associées:

$$
\begin{gathered}
\underline{\varepsilon}^{p}=\lambda \frac{\partial f}{\partial \underline{\underline{\sigma}}} \\
x_{k}=-\lambda \frac{\partial f}{\partial \mathrm{X}_{k}}
\end{gathered}
$$

Ce formalisme permet ainsi de définir les variables d'écrouissage dans un cadre thermodynamique cohé. rent.

\section{ELASTICITÉ}

Lors de sollicitations monotones les déformations élastiques sont souvent négligeables par rapport aux déformations plastiques. Cependant lors de sollicitations cycliques les deux types de déformations peuvent être du même ordre de grandeur, dans ce cas une modélisation réaliste des déformations élastiques s'avère tout à fait essentielle.

Les résultats expérimentaux montrent la nécessité d'une modélisation de type non linéaire.

Une approche de type incrémental a été choisie, ce qui se traduit par l'expression suivante:

$$
\varepsilon^{e}{ }_{y}=\frac{s_{\mathrm{il}}}{2 \mathrm{G}}+\frac{\dot{1}_{1}}{9 \mathrm{~K}} \delta_{\mathrm{i}}
$$

$\mathrm{G}$ et $\mathrm{K}$ sont les modules volumique et de cisaillement que nous proposons d'exprimer par les relations suivantes:

$$
\begin{gathered}
\mathrm{G}=\mathrm{G}_{\mathrm{o}}\left(\frac{\mathrm{I}_{1}}{3 \mathrm{P}_{\mathrm{a}}}\right)^{\mathrm{n}} \\
\mathrm{K}=\mathrm{K}_{\mathrm{o}}^{\mathrm{e}}\left(\frac{\mathrm{I}_{1}}{3 \mathrm{P}_{\mathrm{o}}}\right)^{\mathrm{n}} \mathrm{A}\left(\mathrm{I}_{l}, \mathrm{~s}_{\mu}\right)
\end{gathered}
$$

$G_{0}$ et $K^{e}$ dépendent de l'état initial, $P a$ est la pression atmosphérique.
Nous faisons l'hypothèse que l'énergie de déformation élastique dépend uniquement de $\mathrm{I}_{1}$ et $\mathrm{s}_{11}$ et donc:

$$
\dot{w}=\sigma_{\eta} \dot{\varepsilon}_{v}^{e}=\frac{\partial w}{\partial \mathrm{I}_{1}} \dot{\mathrm{I}}_{1}+\frac{\partial \mathrm{w}}{\partial \mathrm{s}_{\|}} \dot{\mathrm{s}}_{\| 1}
$$

avec nécessairement:

$$
\frac{\partial^{2} \mathrm{w}}{\partial \mathrm{I}_{1} \partial \mathrm{s}_{\mathrm{ul}}}=\frac{\partial^{2} \mathrm{w}}{\partial \mathrm{s}_{\mathrm{u}} \partial \mathrm{I}_{1}}
$$

Les relations (9) à (12) permettent de définir la fonction A qui doit de plus satisfaire à la condition $A\left(I_{1}, 0\right)=1$

$$
A\left(I_{1}, s_{11}\right)=\frac{1}{\left(1-\frac{9 n k_{0}^{e} s_{I I}^{2}}{4 G_{0} I_{1}^{2}}\right)}
$$

Finalement :

$$
K=\frac{K_{o}^{e}\left(\frac{I_{1}}{3 P_{a}}\right)^{n}}{1-\frac{9 n k_{o}^{e} s_{l 1}^{2}}{4 G_{a} l_{1}^{2}}}
$$

Cette approche peut ne pas satisfaire les principes de la thermodynamique pour certains chemins de contraintes (LORET, 1981), ce qui est tout à fait classique pour les modèles de type hypoélastique. Pour remédier à ce problème il faudrait utiliser un modèle élastique complètement cohérent sur le plan thermodynamique et défini à partir de la relation (4).

Cependant dans la plupart des cas usuels, le modèle présenté ici est plus simple à mettre en œuvre et s'avère suffisant.

\section{PLASTICITÉ}

Dans ce modèle deux types (ou mécanismes) de déformations plastiques ont été pris en compte:

- les dêformations $\varepsilon_{v}^{\text {p }}$ dépendant de la variation de contrainte moyenne $\left(\mathrm{I}_{1} / 3\right)$;

— les déformations $\varepsilon^{\text {dp }}$ "dépendant de la variation de $s_{i} / I_{1}$.

La déformation plastique totale s'écrit alors :

$$
\varepsilon^{p}=\varepsilon^{d p}+\frac{1}{3} \varepsilon^{i p}, \delta_{i j}
$$

$\varepsilon^{i p}$ est une déformation isotrope qui sera calculée en utilisant un mécanisme d'écrouissage isotrope dépendant de la variable scalaire q (voir 4.1)

$\varepsilon^{d p}$ comprend deux parties: la partie déviatoire $e^{d p}$ et la partie isotrope $\varepsilon^{d p}$ ", cette déformation est calculée en 
prenant en compte un mécanisme d'écrouissage isotrope (variable p) et un mécanisme d'écrouissage cinématique (variable $\alpha_{i}$ ).

Dans ce cas l'énergie libre et la dissipation s'écrivent:

$$
\begin{aligned}
& \mathrm{W}=\mathrm{W}_{1}+\mathrm{W}_{D}=\mathrm{W}_{\mathrm{e}}\left(\varepsilon^{e}{ }_{v}\right) \\
& +W_{d}(q)+W_{d}\left(p, \alpha_{t}\right) \\
& \emptyset=\emptyset_{1}+\emptyset_{\mathrm{D}}=\underline{\underline{\sigma}}: \dot{\varepsilon}-\dot{\mathrm{W}}
\end{aligned}
$$

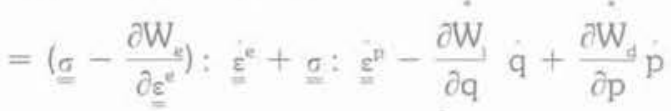

$$
\begin{aligned}
& \left.+\frac{\partial W_{d}}{\partial \underline{\alpha}}: \underline{\alpha}\right) \geqslant 0
\end{aligned}
$$

avec :

$$
\begin{aligned}
& \emptyset_{1}=\underset{\sigma}{\sigma}: \underset{\underline{\underline{\varepsilon}}}{\underline{p}}-\frac{\partial \mathrm{W}}{\partial \mathrm{q}} \dot{q} \geqslant 0 \\
& \emptyset_{\mathrm{D}}=\underline{\sigma}: \underline{\varepsilon}^{\mathrm{dp}}-\frac{\partial \mathrm{W}_{\mathrm{d}}}{\partial \mathrm{p}}-\frac{\partial \mathrm{W}_{\mathrm{d}}}{\partial \underline{\underline{\alpha}}}: \underline{\underline{\alpha}} \geqslant 0
\end{aligned}
$$

\subsection{Mécanisme isotrope $\varepsilon^{i p}{ }_{i j}$}

Les hypothèses suivantes ont été prises en compte:

- la surface de charge

$$
f_{1}=I_{1} / 3-Q=0
$$

- l'évolution de la surface de charge est due à l'évolution de la force thermodynamique $\mathrm{Q}$ associée à la variable d'écrouissage isotrope q.

L'équation (18) peut alors être écrite sous la forme:

$$
\begin{aligned}
\phi_{1}=\sigma_{i j} \varepsilon_{0}-\frac{\partial W_{i}}{\partial q} \dot{q}=\frac{1}{3} I_{1} & \varepsilon^{i p}{ }_{0} \\
& -Q q \geqslant 0
\end{aligned}
$$

avec $Q=\frac{\partial \dot{W}}{\partial \mathrm{q}}$

Les lois d'évolution s'écrivent alors :

$$
\begin{aligned}
& \varepsilon^{i p}=\lambda \frac{\partial f_{i}}{\partial a_{i j}} \Rightarrow \varepsilon_{j}^{i p}=\lambda \\
& \dot{q}=-\lambda \frac{\partial f_{i}}{\partial Q} \Rightarrow \dot{q}=\lambda
\end{aligned}
$$

Les relations (22) et (23) permettent de définir le paramètre d'écrouissage q :

$$
\mathrm{q}=\varepsilon^{\mathrm{p}}
$$

Pour définir complètement ce mécanisme il faut choisir une forme analytique représentative de la fonction $\mathrm{Q}(\mathrm{q})$ :

$$
\mathrm{Q}=\mathrm{Q}_{0} \mathrm{q}^{\mathrm{m}}
$$

Sous forme incrémentale cette relation s'écrit:

$$
\mathrm{Q}=\mathrm{K}_{\mathrm{p}} \dot{\mathrm{q}}
$$

avec :

$$
K_{p}=K_{o}^{p}\left(\frac{Q}{P_{a}}\right)^{n}
$$

ou $\mathrm{K}^{p}$ et $\mathrm{n}$ peuvent être aisément déterminés à partir de résultats expérimentaux.

\subsection{Mécanisme déviatoire $\varepsilon^{\mathrm{dp}}{ }_{\mathrm{ij}}$}

Par soucis de simplification nous présentons tout d'abord (4.2.1) le calcul de $\varepsilon^{d p}$ sans prendre en compte le couplage entre sa partie isotrope $\left(\varepsilon^{d p}\right)$ et sa partie déviatoire $\left(e^{\mathrm{dp}}\right)$. Dans une deuxième partie (4.2.2) nous présenterons le couplage sous la forme d'une condition cinématique qui conduit à une expression modifiée pour $\varepsilon^{d p}$. Cette condition a été introduite pour prendre en compte correctement les variations de volume qui apparaissent lors de sollicitations déviatoires.

\subsubsection{Calcul de $\varepsilon^{d p}$ ij sans prendre en compte le couplage $\varepsilon^{d p}{ }_{v} / e^{d p}{ }_{i j}$}

Les hypothèses suivantes ont été prises en compte (JAFARI, CAMBOU, 1987)

Surface de charge:

$$
\begin{aligned}
f\left(s_{i}, X_{i}, R\right)=q_{\mu} h(\theta)- & R I_{I} \\
& =f_{0}\left(q_{j}\right)-R_{i}
\end{aligned}
$$

où

$$
h(\theta)=(1-\gamma \cos 3 \theta)^{1 / 3}
$$

avec $\theta$ défini (fig. 5) par :

$$
\begin{aligned}
\cos 3 \theta & =2^{1 / 2} 3^{3 / 2} \frac{\operatorname{det} \mathrm{q}}{\mathrm{q}^{3} \pi} \\
\mathrm{q}_{i j} & =\mathrm{s}_{i}-\mathrm{I}_{1} \mathrm{X}_{t j} \\
\mathrm{q}_{\| l} & =\sqrt{\mathrm{q}_{i j} \mathrm{q}_{i j}} \\
\mathrm{q}_{m} & =\sqrt{\operatorname{det} \mathrm{q}}
\end{aligned}
$$

L'expression de la surface de charge (27) peut aussi être exprimée en fonction des invariants de $\mathrm{q}_{40}$ sous la forme:

$$
\mathrm{f}=\left(\mathrm{q}_{u}^{3}-\gamma 2^{1 / 2} 3^{3 / 2} \mathrm{q}^{3}{ }_{u I}\right)^{1 / 3}-\mathrm{Rl}_{1}
$$


L'introduction du paramètre $\gamma$ permet de décrire la dissymétrie du comportement des sols en compression et extension (fig. 3).

Les paramètres $\mathrm{R}$ et $\mathrm{X}$ sont les forces thermodynamiques associés aux paramètres d'écrouissages dans le formalisme des matériaux standard généralisés. Ils mesurent d'une part le gonflement (écrouissage isotrope), d'autre part la translation (écrouissage cinématique) de la surface de charge (fig. 5).

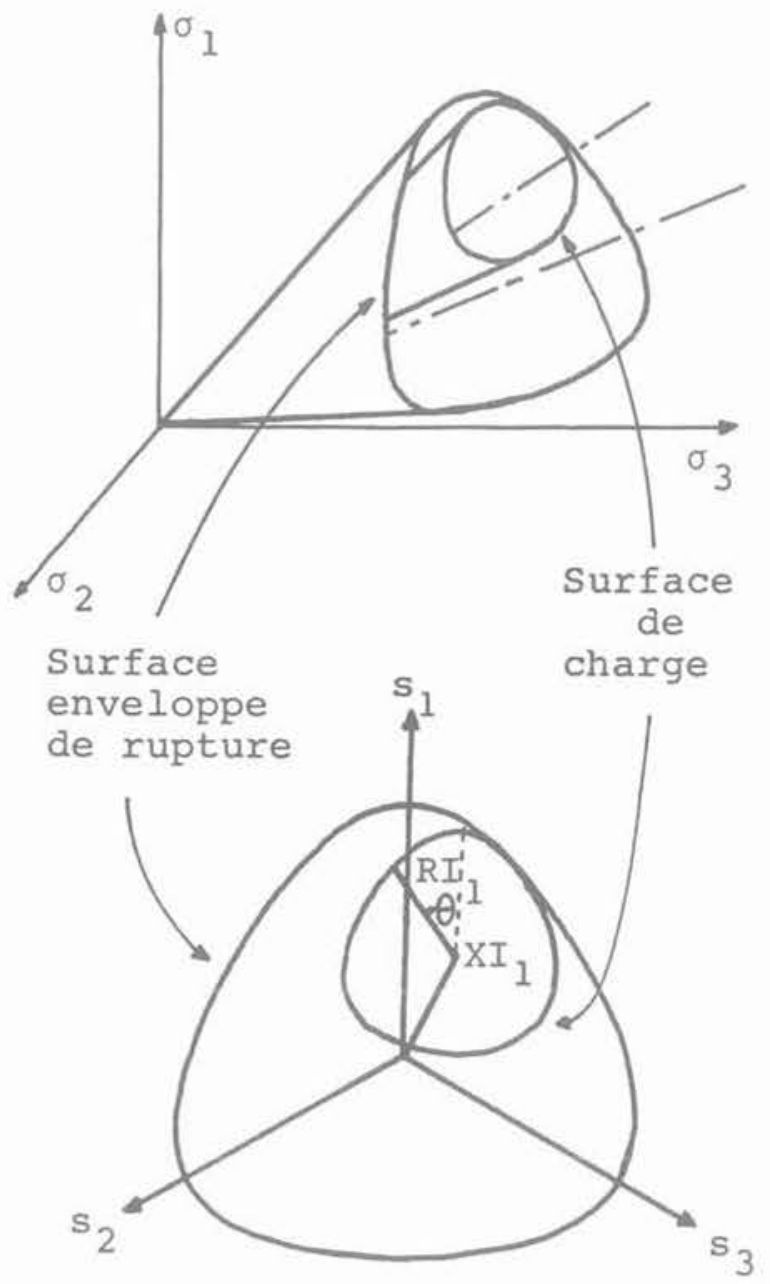

Fig. 5. - Définition de la surface de charge et de la surface enveloppe de rupture.

Fig. 5. - Definition of the yield surface and of the failure surface.

La prise en compte d'un mécanisme d'écrouissage cinématique permet de décrire de façon réaliste le comportement sous sollicitation cyclique ainsi que l'anisotropie induite.

L'évolution de l'état du matériau est décrite par les variables décrouissage $p$ et $\alpha$. L'analyse des résultats expérimentaux obtenus lors d'essais triaxiaux cycliques nous a conduit à conclure à la nécessité de prendre en compte un écrouissage cinématique non linéaire (l'écrouissage linéaire conduisant à une modélisation peu réaliste). De plus ce type d'écrouissage permet de définir la rupture du matériau de façon simple sans introduire la notion de surface de rupture.

La rupture se produit lorsque les paramètres d'écrouis. sage atteignent leurs valeurs limites.

Les deux mécanismes d'écrouissage seront définis par les fonctions $R(p)$ et $X_{y}\left(\alpha_{i}\right)$.

Nous avons considéré ces fonctions sous la forme suivante :

$$
\dot{\mathrm{R}}=\mathrm{g}(\mathrm{p}) \dot{\mathrm{p}}
$$

avec

$$
\begin{gathered}
g(p)=\frac{A}{(1+B p)^{2}} \\
X_{v}=a \dot{\alpha}_{j}
\end{gathered}
$$

La relation (33) semblerait indiquer la prise en compte d'un écrouissage cinématique linéaire, contrairement à ce qui a été annoncé plus haut. En fait la non linéarité va apparaître dans l'écriture de la loi d'évolution de a (relation 36). Cete formulation a été proposée par $\mathrm{CHABOCHE}$ (LEMAITRE et CHABOCHE, 1985).

Les lois d'évolution s'écrivent alors :

$$
\begin{gathered}
\varepsilon^{\mathrm{dp}}=\lambda \frac{\partial \mathrm{f}}{\partial \sigma_{\theta}} \\
\dot{\mathrm{p}}=-\lambda \frac{\partial \mathrm{f}}{\partial \mathrm{R}} \\
\dot{\alpha}_{v}=-\lambda\left(\frac{\partial \mathrm{f}}{\partial \mathrm{X}_{i}}+1_{1} \emptyset \mathrm{X}_{v}\right)
\end{gathered}
$$

Si l'on définit $\mathrm{Q}_{i j}$ par:

$$
\mathrm{Q}_{i j}=\frac{\partial \mathrm{f}_{o}}{\partial \mathrm{q}_{i}}=\frac{1}{\mathrm{~h}^{2}(\theta)}\left(\frac{\mathrm{q}_{u}}{\mathrm{q}_{\|}}-\gamma \frac{\cos 3 \theta}{3} \mathrm{q}_{u} \mathrm{q}^{\mathrm{d}}\right)
$$

$\mathrm{f}_{\text {a }}$ a été défini dans (27) et $\mathrm{q}^{\mathrm{d}}$ signifie : partie déviatoire de $\left(\mathrm{q}_{4}\right)^{-1}$

On peut alors exprimer:

$$
\frac{\partial f}{\partial \mathrm{X}_{i j}}=\frac{\partial \mathrm{f}_{0}}{\partial \mathrm{X}_{i}}=-\mathrm{I}_{1} \mathrm{Q}_{i j}
$$

Les lois d'évolution (34) à (36) s'expriment alors par:

$$
\begin{gathered}
\varepsilon^{\mathrm{dp}}=\lambda \frac{\partial \mathrm{f}}{\partial \sigma_{i j}} \\
\dot{\mathrm{p}}=\lambda \mathrm{I}_{1} \\
\dot{\alpha}_{i j}=\mathrm{I}_{3}\left(\mathrm{Q}_{i j}-\emptyset \mathrm{X}_{i j}\right)
\end{gathered}
$$


avec :

$$
\lambda=\frac{\frac{\partial \mathrm{f}}{\partial \underline{\underline{\sigma}}}: \underline{\underline{\sigma}}}{\mathrm{I}_{1}^{2}[\mathrm{aQ}:(\underline{\underline{Q}}-\phi \mathrm{X})+\mathrm{g}(\mathrm{p})]}
$$

La rupture se produit pour $\lambda$ infini, donc pour $g(p)=0$ et $\mathrm{Q}-\emptyset \mathrm{X}=0$. Les valeurs que prennent alors $\mathrm{X}$ et $\mathrm{R}$ sont les valeurs limites $\mathrm{X}_{\text {lim }}$ et $\mathrm{R}_{\text {lim }}$.

L'enveloppe limite de rupture est la surface enveloppe de toutes les surfaces de charge définies par $\mathrm{X}_{\text {bim }}$ et $\mathrm{R}_{\text {lim }}$

Nous avons supposé que cette surface limite avait une forme semblable à la forme des surfaces de charge. Si nous supposons de plus que lors de la rupture $s_{4}$ et $\mathrm{X}_{\mathrm{u}}$ ont les mêmes directions principales; il suffit de définir $\emptyset$ par la relation suivante :

$$
\emptyset=\emptyset_{0} \mathrm{~h}(\theta) \mathrm{Q}_{I I}
$$

avec :

$$
\mathrm{Q}_{\|}=\sqrt{\mathrm{Q}_{i j} \mathrm{Q}_{0}}
$$

Si $\emptyset_{0}$ est supposé constant le critère de rupture est un cône et dans le plan de Mohr une droite. La courbure du critère de rupture (fig. 6) peut être prise en compte en considérant que $\emptyset$ dépend du premier invariant du tenseur des contraintes $l_{1}$ :

$$
\emptyset_{\mathrm{o}}=\emptyset_{1} \frac{\mathrm{I}_{1}}{3 \mathrm{~Pa}}+\emptyset_{2}
$$

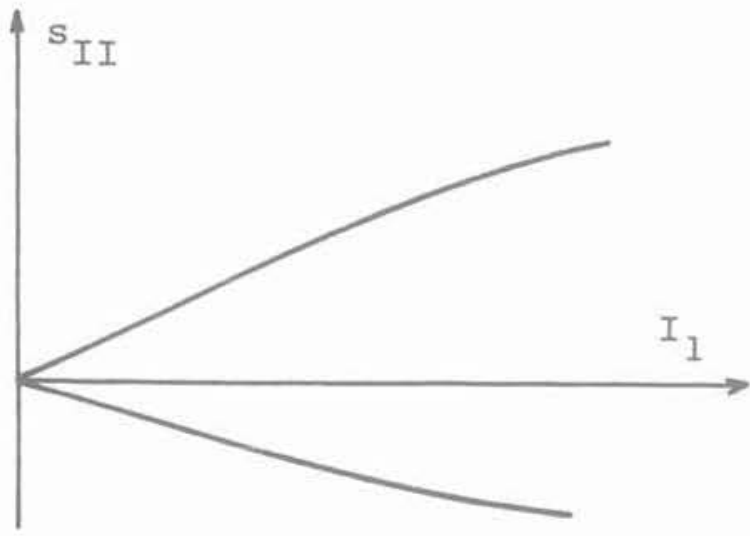

Fig. 6. - Forme de la surface de charge dans le plan $s_{11}$

Fig. 6. - Shape of the yield surface in the axes $s_{\| 1}, h_{1}$

\subsubsection{Calcul de $\varepsilon^{d p}$ en prenant en compte le couplage $\varepsilon^{d p}{ }_{v} / e^{d p}{ }_{i j}$}

Dans les matériaux granulaires, une variation de volume significative peut se produire pour un chargement purement déviatoire. Cette variation de volume est liée à l'aspect discontinu du matériau et aux conditions cinématiques qui en résultent lors du chargement.
Cette « physique » particulière ne permet pas de définir les déformations à partir de la seule relation de normalité (39).

Pour avoir une modélisation correcte des variations de volume nous proposons donc une condition cinématique sous la forme suivante:

$$
\varepsilon_{v}^{d p}=\beta\left(\frac{S_{H I}}{S_{I / c}}-1\right) \frac{\left|S_{i j} e^{d p}{ }_{\|}\right|}{S_{H}}
$$

$\beta$ est une constante à déterminer expérimentalement.

$$
\mathrm{s}_{I l c}=\sqrt{\mathrm{s}_{\hat{\theta}} \mathrm{s}^{c}}
$$

$\mathrm{s}^{c}$ est létat de contrainte associé à $\mathrm{s}_{\|}$par un chemin radial (fig. 7) sur la surface caractéristique.

La surface caractéristique qui est une généralisation de la notion de ligne caractéristique qui a été introduite par LUONG (LUONG, 1980) est définie par:

$$
f_{c}=s_{l l c} h(\theta)-R_{c} I_{1}
$$

La fonction $h(\theta)$ est identique a celle définie pour la surface de charge (28) et (29).

Cette surface caractéristique sépare l'espace des contraintes en un domaine où se produira une contractance et un domaine ou se produira une dilatance (fig. 7), elle est indépendante de la densité initiale du matériau.

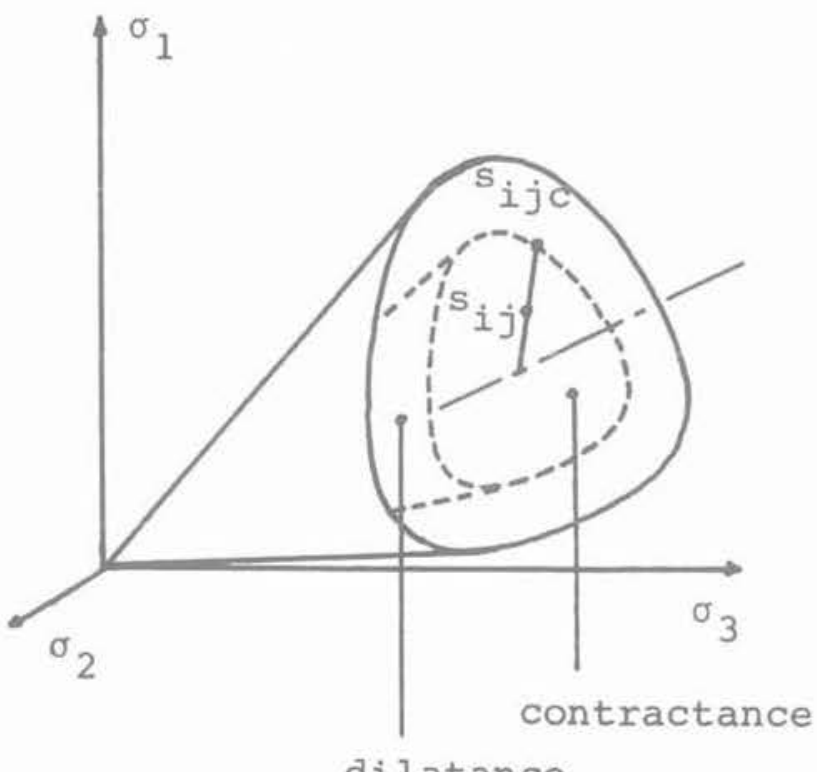

Fig. 7. - Définition de la surface caractéristique.

Fig. 7. - Definition of the characteristic surface.

La relation (44) peut aussi se mettre sous la forme:

$$
\begin{aligned}
& \varepsilon_{v}^{d p}-\beta \frac{s_{\| I}}{s_{\| l c}}-1 \frac{\left|s_{i j} e^{d p}{ }_{j i}\right|}{s_{l l}} \\
& =\mathrm{n}_{t} \varepsilon^{d p}=0
\end{aligned}
$$


avec

$$
\begin{gathered}
n_{i j}=\frac{\beta^{\prime} \frac{s_{i j}}{s_{\| I}}-\delta_{i j}}{\sqrt{\beta^{\prime 2}+3}} \\
\beta^{\prime}=\text { signe de }\left(s_{i j} \varepsilon^{d p}\right) \beta\left(\frac{s_{\| l}}{s_{l i c}}-1\right)
\end{gathered}
$$

La relation (47) impose que $\varepsilon^{\text {dp }}$ "soit normal à $n_{0}$. Donc pour satisfaire la condition cinématique (44) il suffit de prendre dans l'espace des incréments de déformations la projection de $\varepsilon^{\mathrm{dp}}$ sur l'hypersurface de normale $\mathrm{n}_{\mathrm{H}}$ (KANATANI, 1982)"

$$
\varepsilon_{\varepsilon_{0}}^{d p}=\lambda\left(\frac{\partial f}{\partial \sigma_{j}}-\frac{\partial f}{\partial \sigma_{k j}} n_{k j} n_{v}\right)
$$

avec :

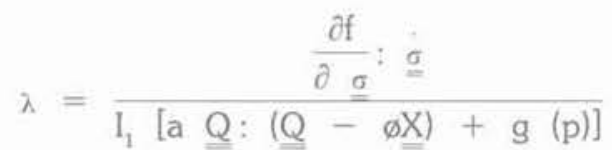

On notera que l'introduction de la condition cinématique (44) qui se traduit par la relation (49) n'est pas compatible avec le formalisme des matériaux standard généralisés. Cependant dans un article précédent nous avons montré (JAFARI et CAMBOU, 1987) qu'un changement de variable sur les contraintes peut permettre de se replacer dans le cadre de ce formalisme. $\mathrm{Ce}$ changement de variable n'entraînant aucune modification sur les résultats nous garderons la présentation cidessus qui est plus simple.

\section{RÉSUMÉ DU MODÈLE DE COMPORTEMENT}

Le modèle proposé peut être résumé par les relations suivantes :

a) Partie élastique:

$$
\varepsilon^{e}=\frac{\dot{s}_{i v}}{2 G}+\frac{\dot{I}_{1}}{9 K} \delta_{i n}
$$

avec :

$$
\begin{gathered}
G=G_{0}\left(\frac{I_{1}}{3 P_{a}}\right)^{n} \\
K=\frac{K_{o}^{e}\left(\frac{I_{1}}{3 P_{a}}\right)^{n}}{1-\frac{9 n K_{o}^{e} s^{2} \pi}{4 G I_{1}^{2}}}
\end{gathered}
$$

$\mathrm{K}_{\mathrm{o}}^{\mathrm{e}}, \mathrm{G}_{\mathrm{o}}$ et $\mathrm{n}$ sont des paramètres à déterminer expérimentalement.

b) Déformation plastique liée à la partie isotrope du tenseur des contraintes.

Critère:

$$
f_{1}=\frac{I_{1}}{3}-Q \leqslant 0
$$

Lois d'évolution :

$$
\begin{gathered}
\varepsilon^{i p}=\dot{q} \\
\dot{Q}=K_{p} \dot{q}
\end{gathered}
$$

avec :

$$
K_{p}=K_{o}^{p}\left(\frac{Q}{P_{a}}\right)^{n}
$$

$\mathrm{K}_{\mathrm{o}}^{\mathrm{p}}$ est un paramètre à déterminer expérimentalement.

c) Déformation plastique liée à la partie déviatoire du tenseur des contraintes.

Critère:

$$
f\left(s_{i j}, X_{u}, R\right)=q_{u} h(\theta)-R_{1}
$$

Lois d'évolution :

$$
\begin{aligned}
& \varepsilon^{d p}=\lambda\left(\frac{\partial f}{\partial \sigma_{j}}-\frac{\partial f}{\partial \sigma_{k l}} n_{k j} n_{j}\right) \\
& \mathrm{p}=\lambda \mathrm{I}_{1} \\
& \mathrm{R}=\mathrm{g}(\mathrm{p}) \mathrm{p} \\
& \dot{\alpha}_{i j}=\lambda_{1}\left(Q_{i j}-\phi X_{i j}\right) \\
& \dot{X}_{\mathrm{y}}=\mathrm{a} \alpha_{\mathrm{u}}
\end{aligned}
$$

avec:

$$
\begin{aligned}
& \lambda=\frac{\frac{\partial f}{\partial \underline{\underline{\sigma}}}: \underline{\underline{\sigma}}}{\mathrm{I}_{1}^{2}[\mathrm{aQ}:(\underline{\underline{Q}-\phi \mathrm{X})}+\mathrm{g}(\mathrm{p})]} \\
& \emptyset=\emptyset_{1} \frac{\mathrm{I}_{1}}{3 \mathrm{P}_{\mathrm{a}}}+\emptyset_{2} \\
& g(p)=\frac{A}{(1+B p)^{2}} \\
& \mathrm{n}_{\mathrm{ij}}=\frac{\beta^{\prime} \frac{\mathrm{s}_{\mathrm{ll}}}{\mathrm{s}_{\mathrm{ll}}}-\delta_{\mathrm{i}}}{\sqrt{\beta^{\prime 2}+3}} \\
& \beta^{\prime}=\text { signe de } s_{V} \varepsilon^{d p} \beta\left(\frac{s_{I I}}{s_{\| k}}-1\right)
\end{aligned}
$$

$\mathrm{A}, \mathrm{B}, \mathrm{a}, \emptyset_{1}, \emptyset_{2}, \beta$ sont des paramètres à déterminer expérimentalement. 
Surface caractéristique :

$$
f_{c}=s_{\mathrm{Hc}} h(\theta)-R_{c} I_{1}
$$

$\mathrm{R}_{\mathrm{c}}$ est un paramètre à déterminer expérimentalement.

\section{Procédure d'identification des paramètres}

Pour déterminer les 12 paramètres de ce modèle, il faut disposer des trois types d'essais suivants :

- un essai de chargement déchargement isotrope;

- un essai triaxial en compression avec des déchargement à différents niveaux;

- un essai triaxial en extension.

Ces essais vont permettre de définir les paramètres du modèle selon la procédure suivante:

\subsection{Essai isotrope}

La courbe de déchargement permet de déterminer les paramètres élastiques $\mathrm{K}^{\mathrm{e}}$ et $\mathrm{n}$ à partir d'une méthode d'ajustement de courbe.

Le paramètre plastique $\mathrm{k}^{p}$ peut être déterminé de façon analogue à partir de la courbe de charge.

\subsection{Essai triaxial en extension}

Cet essai est utilisé pour déterminer le paramètre $\gamma$ qui apparaît dans le critère déviatoire.

Pour un chargement triaxial en compression ce critère s'écrit:

$$
\sqrt{3 / 2}\left|\mathrm{~s}_{11}\right|(1-\gamma)^{1 / 3}-\mathrm{RI}_{1}=0
$$

Pour un essai triaxial en compression le critère s'écrit:

$$
\sqrt{3 / 2}\left|\mathrm{~s}_{11}\right|(1+\gamma)^{1 / 3}-\mathrm{RI}_{1}=0
$$

et donc :

$$
\left(\frac{1-\gamma}{1+\gamma}\right)^{1 / 3}=\frac{\left(\sigma_{33}-\sigma_{11}\right)\left(\sigma^{c}{ }_{11}+2 \sigma_{33}\right)}{\left(\sigma^{e}{ }_{11}-2 \sigma_{33}\right)\left(\sigma^{c}{ }_{11}-\sigma_{33}\right)}
$$

$\sigma_{11}^{c}$ est la valeur limite obtenue en compression et $\sigma_{11}^{e}$ en extension.

\subsection{Essai triaxial en compression}

- Détermination des paramètres $R$ et $\beta$ à partir de la courbe de variation de volume. Si la valeur de la contrainte axiale correspondant à une variation de volume nulle est notée $\sigma^{\circ}$ (fig. 8), la définition de la surface caractéristique (68) conduit à :

$$
R_{c}=\frac{\sigma^{0}{ }_{11}-\sigma_{33}}{3\left(\sigma_{11}^{0}+2 \sigma_{33}\right)}(1-\gamma)^{1 / 3}
$$

La condition cinématique écrite sous la forme (44) permet de déterminer $\beta$ lors de cet essai :

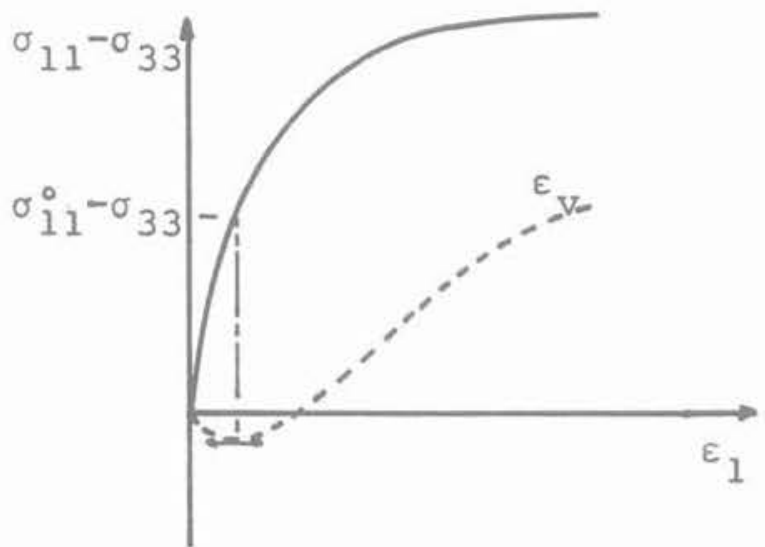

Fig. 8. - Définition de $\sigma^{\circ}$ i1 pour la détermination de $\beta$ et $R_{\mathrm{c}}$.

Fig. 8 - Definition of $\sigma_{11}^{\circ}$ for the calculation of parame. ters $\beta$ and $R_{\mathrm{c}}$.

$$
\beta=\frac{\varepsilon^{d p}}{e^{d p}} 2 \sqrt{\frac{2}{3}} \frac{\sigma^{\circ}{ }_{11}-\sigma_{33}}{\sigma_{11}-\sigma_{11}^{\circ}}
$$

- Identification des paramètres $A, B, \emptyset_{1}, \emptyset_{2}$, a définissant les 2 mécanismes d'écrouissage caractérisant $\varepsilon^{d p}$

Les essais triaxiaux sont menés avec des décharges à différents niveaux (fig. 9).

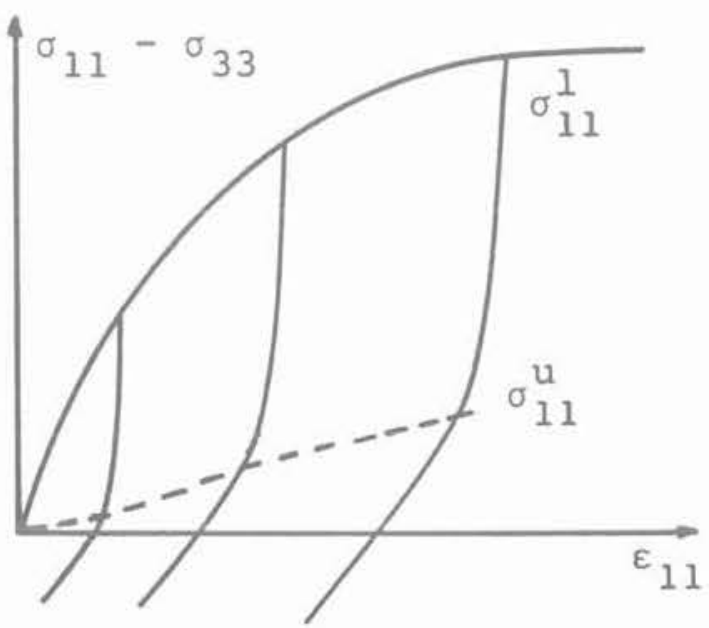

Fig. 9. - Définition de $\sigma_{11}$ et $\sigma_{11}{ }_{11}$ pour la détermination $\operatorname{de} A, B, \theta_{1}, O_{2}, a$.

Fig. 9. Definition of $\sigma_{11}^{2}$ and $\sigma_{11}^{2}$ for the calculation of parameters $A, B, \emptyset_{1}, \emptyset_{2}, a$. 
Les limites du * domaine élastique " sont notées $\sigma^{1}$ (chargement) et $\sigma^{\mathrm{u}}$ (déchargement), ces valeurs satisfont le critère (57) donc:

$$
\begin{aligned}
& \sqrt{\frac{3}{2} s_{11}^{1}-\mathrm{P}_{1} \mathrm{X}_{11}(1-\gamma)^{1 / 3}-\mathrm{RI}_{1}^{\prime}}=0 \\
& \sqrt{\frac{3}{2} s_{11}^{u}-\mathrm{I}_{1}^{u} \mathrm{X}_{11}(1+\gamma)^{1 / 3}-\mathrm{RI}_{1}^{u}}=0
\end{aligned}
$$

Pour chaque niveau de déchargement les 2 relations précédentes permettent de définir les valeurs de $X_{11}$ et $\mathrm{R}$.

De plus la loi d'évolution du paramètre p peut s'écrire:

$$
\dot{\mathrm{p}}=\lambda \mathrm{I}_{1}
$$

La valeur de $\lambda$ étant définie par la relation 63 il sera donc possible de déterminer pour chaque niveau de déchargement les valeurs de $\mathrm{R}, \mathrm{X}$, et $\mathrm{p}$ et donc d'avoir une représentation approchée des fonctions $R(p)$ et $\mathrm{X}(\mathrm{p})$.

Nous avons supposé que la fonction $\mathrm{R}(\mathrm{p})$ était de la forme :

$$
R(p)=\frac{A p}{1+B p}
$$

$A$ et $B$ peuvent alors être aisément calculés (fig. 10).

Pour les autres paramètres on a :

$$
\dot{X}_{11}=a \alpha_{11}=a l_{1}\left(Q_{11}-\varnothing X_{11}\right)
$$

donc :

$$
\frac{\dot{X}_{11}}{\dot{p}}=a\left(Q_{11}-\phi X_{11}\right)
$$

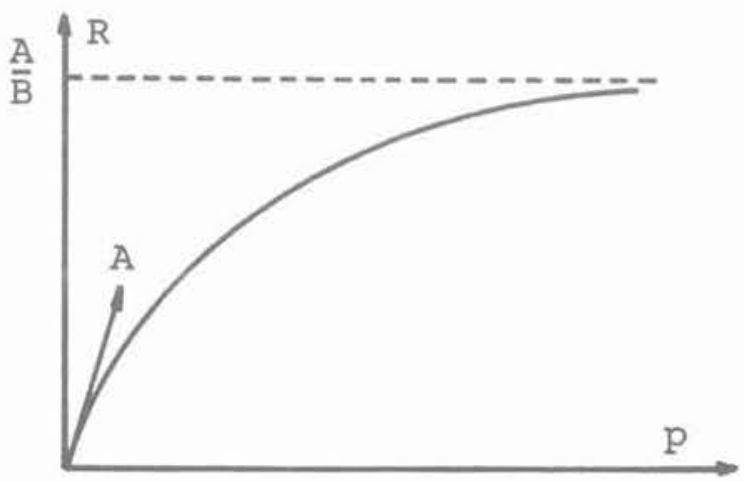

Fig. 10. - Détermination de $A$ et $B$. Fig. 10. - Calculation of $A$ and $B$
$\left(\mathrm{X}_{11} / \mathrm{p}\right)$ est la pente de la courbe $\mathrm{X}(\mathrm{p})$ (fig. 11) que l'on peut donc déterminer et tracer en fonction de $\mathrm{X}_{11}$ fig. 12). $\varnothing$ et a sont alors facilement déterminés. Si cette évaluation est obtenue pour différentes valeurs de $\sigma_{3}$, on pourra donc déterminer les valeurs de $\emptyset_{1}$ et $\emptyset_{2}$ apparaissant dans l'expression (64).

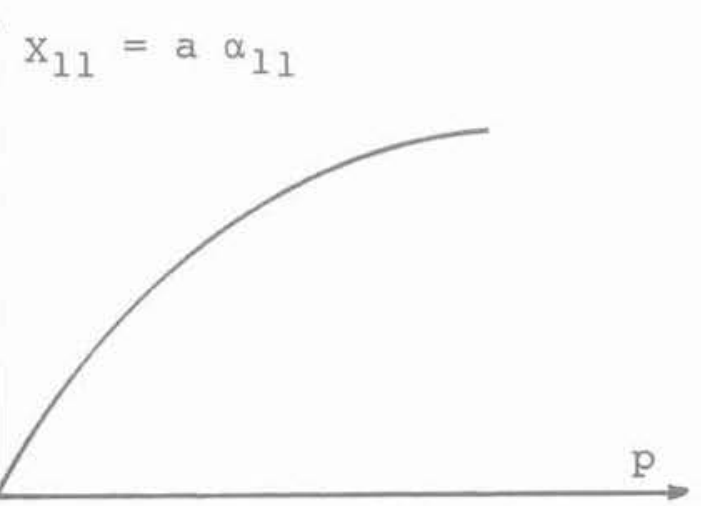

Fig. 11. - Courbe $X_{11}(p)$ utilisée dans la détermination de $a$ et 0 .

Fig. 11. - Curve $X_{11}$ (p) used for the calculation of a and $\emptyset$.

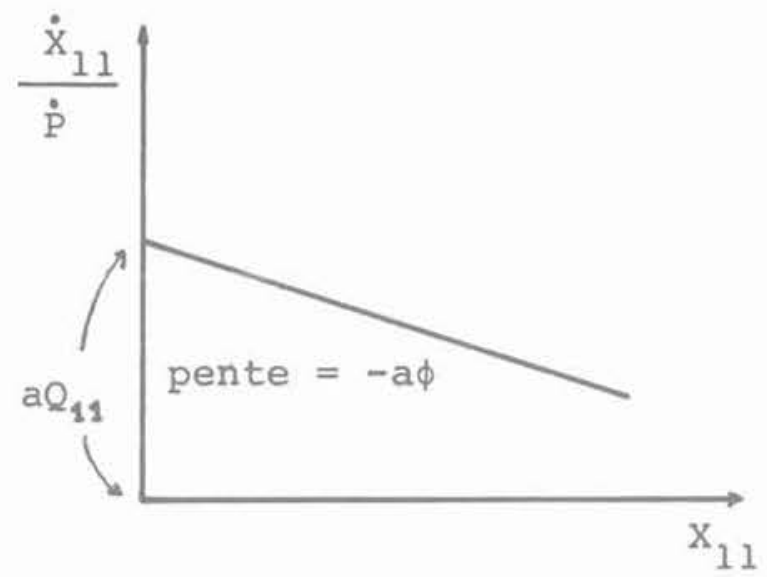

Fig. 12. - Détermination de $a$ et $\mathbf{z}$.

Fig. 12. - Calculation of a and .

La procédure qui vient d'être décrite peut paraître complexe, on notera cependant:

a) que l'expérience acquise après plusieurs identifications permet de traiter une procédure sans trop de difficulté et assez rapidement.

b) que l'expérience acquise après plusieurs indentifications permet de définir un ordre de grandeur acceptable pour certains paramètres difficiles à identifier si l'on ne dispose pas de toutes les données nécessaires (décharges à plusieurs niveaux par exemple).

c) qu'une procédure d'identification automatique est en cours d'élaboration. 


\section{EXEMPLE D'IDENTIFICATION \\ ET DE VALIDATION SUR CHEMINS \\ DE SOLLICITATION HOMOGËNE}

Pour analyser la validité d'un modèle de comportement, il existe deux types de validations:

a) des prévisions réalisées alors que l'on connait les résultats expérimentaux;

b) des prévisions réalisées alors que l'on ne connaît pas les résultats expérimentaux.

Les simulations de types a) sont très utiles lors de la mise au point du modèle mais seules les simulations de types b) peuvent permettre de tester réellement sa validité

Quelques simulations du type a) ont été proposées dans un précédent article (JAFARI, CAMBOU, 1987). Nous présenterons ici des simulations du type b).

Ces simulations sont extraites des prévisions demandées pour le Workshop qui a eu lieu à Cleveland (USA) en

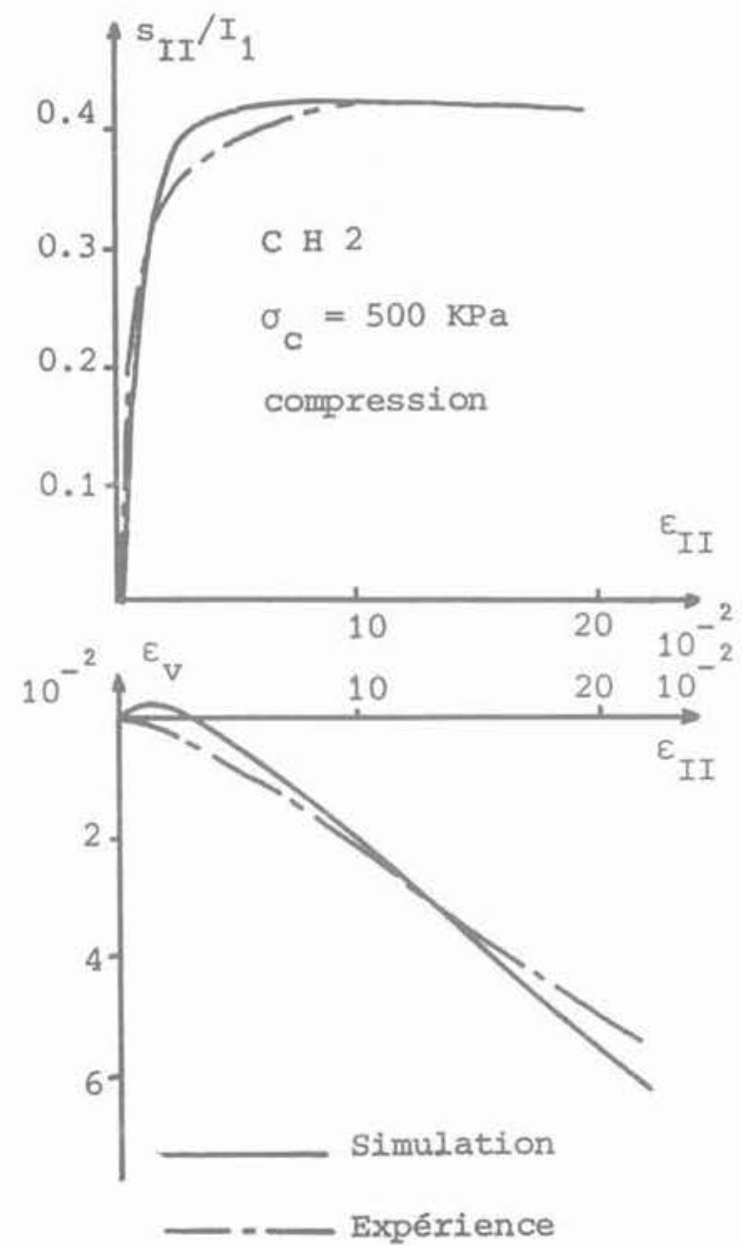

Fig. 13. - Essai triaxial axisymétrique utilisé pour l'identification des paramètres du modèle pour le sable d'Hostun R.F.

Fig. 13. - Axisymetric triaxal test used to calculate the model parameters for the Hostun sand.
Juillet 1987 (International Workshop on constitutive equations for granular non cohesive soils).

La figure 13 présente l'un des essais utilisé pour la détermination des paramètres du modèle.

Les paramètres du modèle obtenu pour le sable d'Hostun ont été les suivants:

$\begin{array}{ll}\mathrm{n}= & 0.600 \\ \mathrm{~K}^{e}= & 49000 \\ \mathrm{~K}^{\mathrm{p}}= & 65000 \\ \mathrm{G}= & 30000 \\ \mathrm{R}= & 0,25 \\ \beta^{\mathrm{c}}=-0,98 \\ \gamma=0,450 \\ \mathrm{~A}=0,015 \\ \mathrm{~B}=0,100 \\ \mathrm{a}=0,015 \\ \emptyset_{1}=0,05 \\ \emptyset_{2}=4,4\end{array}$

La première simulation (fig. 14) correspond à un essai de compression torsion réalisé sur un échantillon ayant la forme d'un cylindre creux. L'essai est caractérisé par un chemin de contrainte radial dans le plan déviatoire, défini par $b=\left(\sigma_{2}-\sigma_{3}\right) /\left(\sigma_{1}-\sigma_{3}\right)=0,286$ (essai noté $\mathrm{HH} 1$ ).

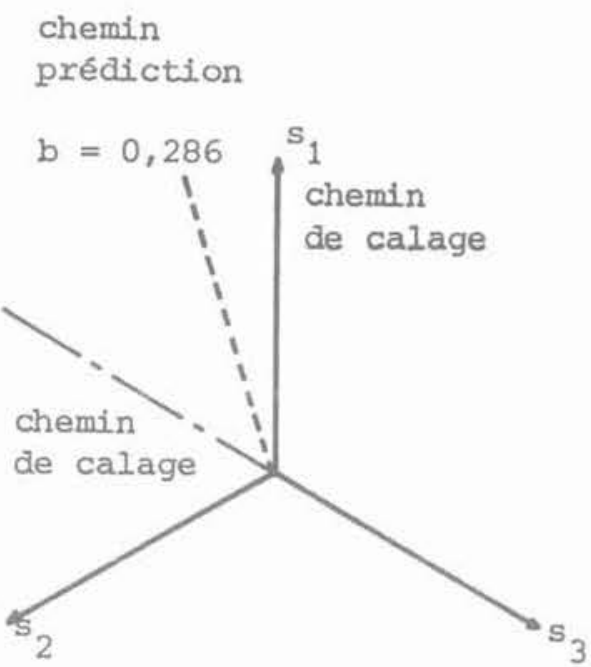

Fig. 14. - Chemin de sollicitation pour la première prévision présentée.

Fig. 14. - Loading path for the first prediction.

La prévision présentée sur la figure 15 apparaît tout à fait correcte tant pour la courbe $\sigma-\varepsilon$ que pour la courbe de variation de volume.

On notera cependant que cette prévision peut être qualifiée de relativement facile car elle correspond à un chemin de sollicitation relativement proche des chemins de calage du modèle (fig. 14).

La deuxième simulation (fig. 16) correspond à un chemin triaxal axisymétrique puis une torsion alternée de 5 cycles et enfin un essai triaxial axisymétrique jusqu'à rupture. Cette prévision peut être qualifiée de difficile car le chemin suivi lors de la torsion est très différent des chemins de calage. Les résultats obtenus (fig. 17) 

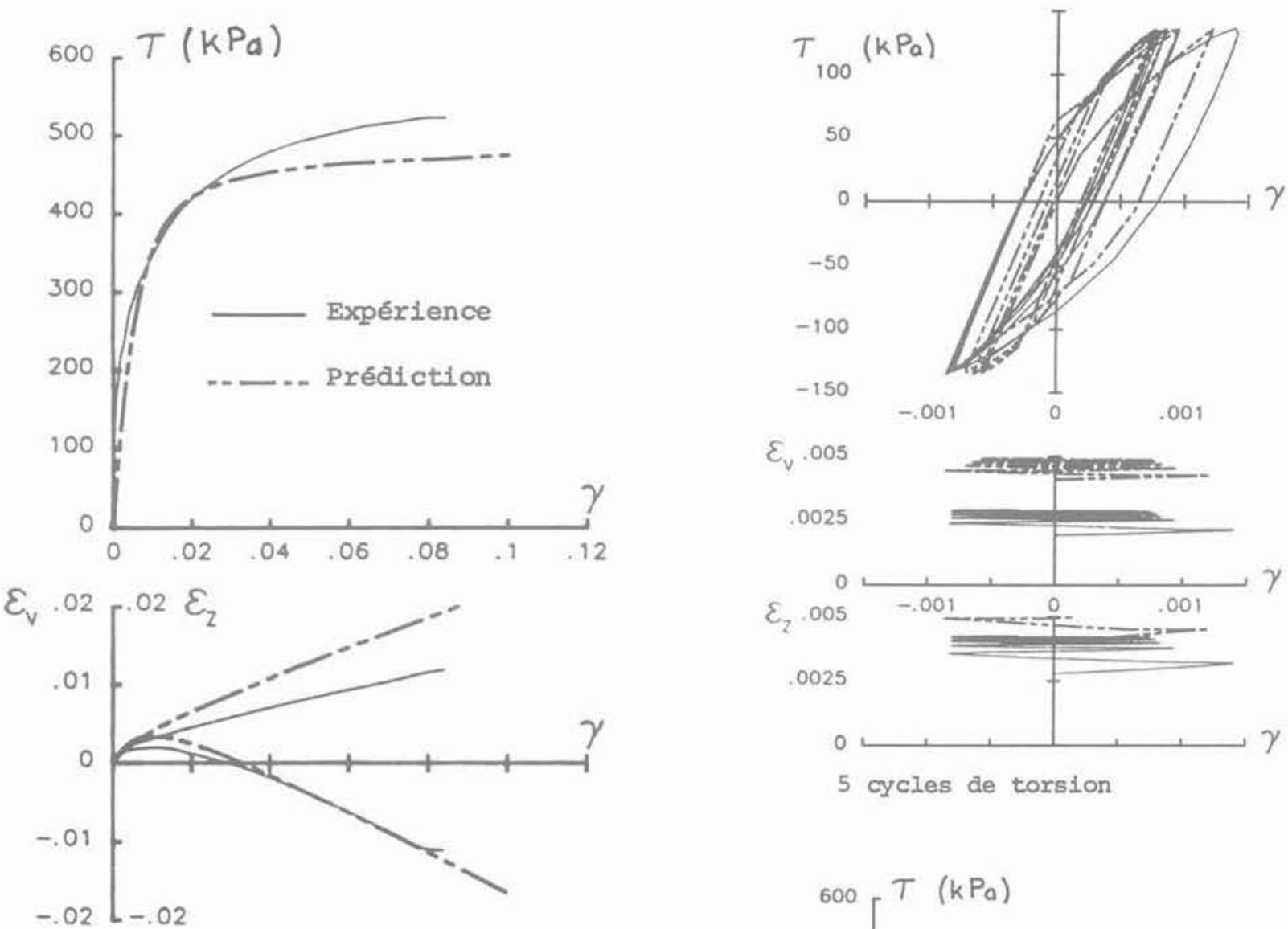

Fig. 15. - Comparaison entre la prévision obtenue par le modèle et les résultats expérimentaux pour la sollicitation définie sur la fig. 14.

Fig. 15. - Comparison between the model prediction and test results for the loading defined on fig 14.

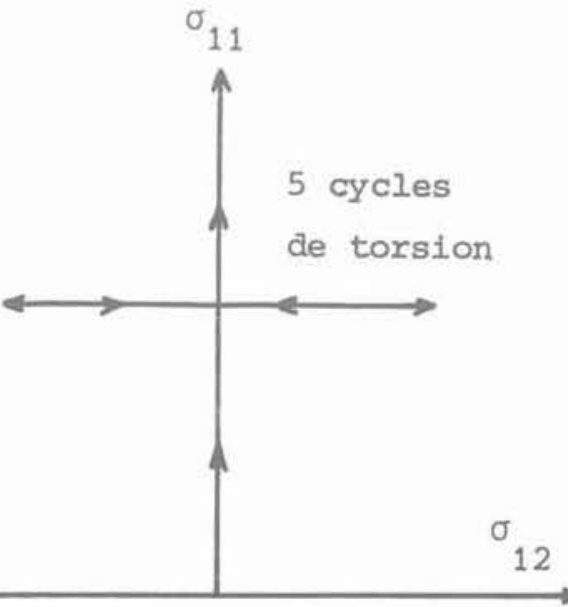

Fig. 16. - Chemin de sollicitation pour la deuxième prévision présentée.

Fig. 16. - Loading path for the second prediction.

Fig. 17. - Comparaison entre la prévision obtenue par le modèle et les résultats expérimentaux pour la sollicitation définie sur la fig. 16.

Fig. 17. - Comparison between the model prediction and test results for the loading defined on fig. 16 .

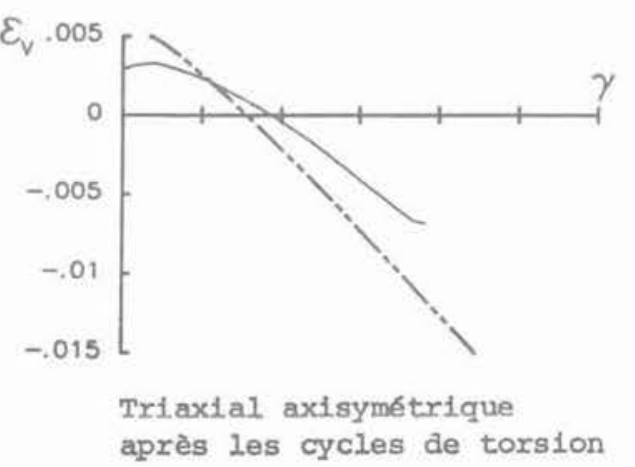


sont très satisfaisants. On notera que lors du Workshop un nombre très faible de modèles a fourni une prévision acceptable pour cette simulation.

Pour être complet on notera que sur l'ensemble des 12 prédictions demandées notre modèle a fourni deux prédictions assez éloignées des résultats expérimentaux pour la simulation d'un chemin cyclique circulaire dans l'espace déviatoire. Cette prédiction difficile n'a été satisfaisante que pour un seul modèle (F. Darve). Pour améliorer ce type de simulation il faudrait affiner les lois caractérisant l'évolution de la surface de charge au cours des sollicitations.

C'est ce à quoi nous nous employons actuellement sur la base des résultats expérimentaux développés dans le cadre du GRECO-CNRS : rhéologie des géomatériaux.

\section{CONCLUSIONS}

- Le modèle de comportement que nous avons présenté est bien adapté pour les matériaux granulaires sans cohésion.

- Il est construit dans un cadre thermodynamique cohérent.

- La condition cinématique introduite dans le modèle permet de décrire de façon simple et réaliste les variations de volume.

- La prise en compte de 3 mécanismes d'écrouissages dont un, cinématique non linéaire permet de décrire de façon satisfaisante l'évolution de l'état du matériau lors de chargements complexes.

- Les simulations réalisées à ce jour permettent d'assurer que ce modèle présente une plage de validité tout à fait intéressante.

\section{BIBLIOGRAPHIE}

1. CAMBOU B. (1979), « Approche du comportement d'un sol considéré comme un milieu non continu ". Thèse présentée à IUniversité de Lyon I, soutenue le 19 mars 1979, pour obtenir le grade de Docteur es Sciences.

2. CAMBOU B., NDEKO S., OLIVARI G., SIDOROFF F. (1983), "Modèle de comportement des sols en tant que matériaux standard généralisés ». Journées Universitaires Génie Civil Nancy.

3. HALPHEN B., NGUYEN Q.S. (1975), «Sur les matériaux standard généralisés ». Journal de Mécanique, Vol. 14, Paris, p. 39-64.

4. JAFARI, K., CAMBOU B. (1987), « Modélisation élastoplastique du comportement des matériaux granulaires $.3^{e}$ joumées Universitaires de Géothechnique, St Nazaire.

5. KANATANI K.I. (1982), « Mechanical foundation of the plastic deformation of granular materials *. IUTAM Conference on deformation and failure of granular materials, Delft, 31 Aug. - 3 sept. P.A. Vermeer, H.J. Luger, A.A. Balkema, Rotterdam, p. $119,127$.

6. LEMAÎTRE J., CHABOCHE J.L. (1985), « Mécanique des matériaux solides ». Dunod, Paris.

7. LORET B. (1986), "Formulation d'une loi de comportement élastoplastique des milieux granulaires ». Thèse présentée à l'Université Paris VI, soutenue le 6 mai 1986, pour obtenir le grade de Docteur es Sciences.

8. LUONG, M.P. (1980) «Phénomènes cycliques dans les sols pulvérulents». Revue Française de Géotechnique, Vol. 10, Paris, p. 39-53. 